\title{
The Jump in Vorticity across a Shock Wave in Relativistic Hydrodynamics
}

\author{
A. V. Gopalakrishna \\ Department of Applied Mathematics, Indian Institute of Science, Bangalore, India
}

Received February 18, 1974; in revised form March 12, 1975

\begin{abstract}
Using the singular surface theory, an expression for the jump in vorticity across a shock wave of arbitrary shape propagating in a uniform, perfect fluid occupying the space-time of special relativity, has been derived. It has been shown that the jump in vorticity across a shock of given strength and curvature depends only on the velocity of the medium ahead of the shock.
\end{abstract}

\section{Introduction}

The study of shock wave propagation in relativistic hydrodynamics is of interest in supernova explosions. The relativistic hydrodynamical equations for one-dimensional motion have been studied by Taub [1], and Johnson and Mckee [2] among others. It will be of interest to study the vorticity generated by a shock in relativistic hydrodynamics.

The jump in vorticity across a shock wave in classical gasdynamics has been studied by Truesdell [3], Lighthill [4], and Hayes [5], among others. Truesdell first obtained a general expression for the vorticity behind a curved two-dimensional steady shock in a uniform flow. Lighthill has generalized Truesdell's result in showing that the result was valid, when expressed in terms of the axes of principal curvature, for a steady shock wave of general shape in a uniform flow. In view of Truesdell's result, namely, "The magnitude of the vorticity generated by a shock of given strength and curvature depends only on the magnitude of the tangential component of velocity and is independent of the form of the equation of state", Hayes derived an expression for the jump in vorticity across a gasdynamic discontinuity without recourse to any thermodynamical law.

The aim of this work is to derive, using the singular surface theory, an expression for the jump in vorticity across a shock wave propagating in a uniform, perfect fluid occupying the space-time of special relativity. We have to note that in relativity, mass and energy are equivalent. Consequently, the energy equation enters into the discussion automatically. However, the expression for the jump in vorticity does not explicitly depend upon the caloric equation of state which characterises the thermodynamical nature of the fluid. It depends upon the strength, defined as the ratio of jump in mass density to the density in front of the shock, the curvature of the shock, and on the velocity in front of the shock (see Section 5). The components of the jump in vorticity have been written in the rest frame of the shock. 


\section{Equations of Motion}

Consider a co-ordinate system $x^{A}$ with $x^{0}=t$ as time and $x^{i}$ as spatial coordinates in the flat space-time of special relativity with the fundamental metric tensor $h_{A B}$ as $h_{00}=c^{2}, h_{i j}=-\delta_{i j}, h_{A B}=0(A \neq B)$, where $c$ is the velocity of light in vacuum. Latin capital indices range over $0,1,2,3$ and the lower case Latin indices assume the values 1, 2, 3 only. Usual summation convention has been followed.

The equations of motion of a perfect fluid in special relativity, described by the stress-energy tensor [6]

$$
T^{A B}=\varrho \mu U^{A} U^{B}-\frac{p}{c^{2}} h^{A B}
$$

can be written as

$$
\begin{aligned}
& \varrho U^{B}\left(\mu U^{A}\right),{ }_{B}=\frac{1}{c^{2}} p,{ }_{B} h^{A B}, \\
& \left(\varrho U^{B}\right){ }_{B}=0,
\end{aligned}
$$

where $\varrho$ is the proper mass density, $p$ is the pressure, $U^{A}$ is the unit, four-dimensional, fluid-velocity vector,

$$
\mu=1+\frac{1}{c^{2}}\left(e+\frac{p}{\varrho}\right)
$$

and

$$
e=e(p, \varrho)
$$

is the proper specific internal energy of the fluid. Equation (2.5) is referred to as the caloric equation of state of the fluid. In the above equations comma followed by a Latin index denotes partial differentiation.

\section{Shock Conditions and Compatibility Conditions}

A shock wave is considered as a propagating, time-like, singular hypersurface $\Sigma$ across which at least some of the field variables describing the fluid motion are discontinuous. It is represented parametrically by the equations

$$
x^{A}=x^{A}\left(u^{\alpha}\right)
$$

where $u^{\alpha}$ (Greek indices assume the values $0,1,2$ ) are the co-ordinates on $\Sigma$. Let $a_{\alpha \beta}$ and $b_{\alpha \beta}$, be respectively, the components of the first, and second fundamental tensors of $\Sigma$. Let $N^{A}$ be the space-like unit normal vector to $\Sigma$. Now let us note the following formulae [7].

$$
\begin{aligned}
& a_{\alpha \beta}=h_{A B} x^{A},_{\alpha} x^{B}, \quad N_{A} N^{A}=-1, \quad N_{A} x^{A},{ }_{\alpha}=0, \\
& x^{A}{ }_{, \alpha \beta}=b_{\alpha \beta} N^{A}, \quad N^{A}{ }_{, \alpha}=b_{\alpha}^{\beta} x^{A}{ }_{, \beta}, \\
& a^{\alpha \beta} x^{A}{ }_{, \alpha} x^{B}{ }_{, \beta}=h^{A B}+N^{A} N^{B}, \quad x_{A, \alpha}=h_{A B} x^{B},{ }_{\alpha} \quad \text { (notation), }
\end{aligned}
$$


where comma followed by a Greek index denotes coveriant derivative with respect to $a_{\alpha \beta}$; since $x^{A}$ are scalar functions of $u^{\alpha}$, we have $x^{A}{ }_{, \alpha}=\partial x^{A} / \partial u^{\alpha}$.

The shock conditions that must be satisfied across $\Sigma$ are [6],

$$
\begin{aligned}
& {\left[T^{A B}\right] N_{B}=0,} \\
& {\left[\varrho U^{B}\right] N_{B}=0,}
\end{aligned}
$$

where $[F]=F_{2}-F_{1}$; the subscript $1(2)$ on $F$ denotes the value of $F$ ahead of (behind) the surface $\Sigma$.

Further, we have the compatibility conditions, derived by using Hadamard's Lemma, which must be satisfied across $\Sigma$ by the partial derivatives of the field variables [8]. For the first partial derivatives, these are

$$
\left[F,,_{A}\right]=\lambda N_{A}+a^{\alpha \beta} v,_{\alpha} x_{A, \beta},
$$

where $[F]=v,\left[F,{ }_{A}\right] N^{A}=-\lambda$ denote the jumps in the field variable $F$ and its normal derivative.

\section{Strength of the Shock and Jumps in $V^{A}$ and $\mu$}

Consider the vector $V^{A}$ and $\tau$ defined by

$$
V^{A}=\mu U^{A} ; \quad \tau=\varrho / \mu .
$$

From (4.1) and (3.4) we get,

$$
M=\tau_{2} V_{2}^{B} N_{B}=\tau_{1} V_{1}^{B} N_{B}=\varrho_{2} U_{2}^{B} N_{B}=\varrho_{1} U_{1}^{B} N_{B} .
$$

By using (4.1), (4.2) and (2.1) the equation (3.3) can be written as

$$
M\left[V^{A}\right]=\frac{1}{c^{2}}[p] N^{A} .
$$

Let us denote the jumps across $\Sigma$ in the pressure $p$, the velocity vector $V^{A}$, the normal derivatives of these and the normal component of $V^{A}$ by

$$
\begin{aligned}
& {[p]=\eta, \quad\left[V^{A}\right]=\lambda^{A}, \quad\left[p,{ }_{B}\right] N^{B}=-\bar{\eta},} \\
& {\left[V^{A},{ }_{B}\right] N^{B}=-\bar{\lambda}^{A}, \quad\left[V^{A}\right] N_{A}=\lambda^{A} N_{A}=-\lambda .}
\end{aligned}
$$

By multiplying Eq. (4.3) by $N_{A}$ and also by $x_{A, \alpha}$ and summing over $A$, we obtain

$$
\begin{aligned}
-M c^{2} \lambda+\eta & =0, \\
{\left[V_{\alpha}\right]=\left[V^{A}\right] x_{A, \alpha} } & =0 .
\end{aligned}
$$

Since Eq. (4.6) means that the tangential component of $V^{A}$ is continuous across the shock, we have

$$
\left[V^{A}\right]=\lambda^{A}=\lambda N^{A} .
$$


By using (4.1) rewrite the Eq. (2.2) in terms of $V^{A}$ and $\tau$. Then take jumps in the resulting equation by using the Eq. (3.5) and the fact that the medium ahead of the shock is uniform and is in constant state, to obtain.

$$
c^{2}\left(M \bar{\lambda}^{A}+\tau_{2} a^{\alpha \beta} \lambda_{,{ }_{\alpha}}^{A} V_{\beta 2}\right)=\bar{\eta} N^{A}+a^{\alpha \beta} \eta,_{\alpha} x_{, \beta}^{A} .
$$

By multiplying (4.8) by $x_{A, \gamma}$ and summing over $A$, we obtain

$$
c^{2}\left(M \bar{\lambda}_{\gamma}+\lambda \tau_{2} V_{2}^{\alpha} b_{\alpha \gamma}\right)=\eta_{, \gamma}
$$

By differentiating Eq. (4.5) tangentially, we get

$$
c^{2}\left(M \lambda_{, \gamma}+\lambda \tau_{1} V_{1}^{\alpha} b_{\alpha \gamma}\right)=\eta_{,_{\gamma}} .
$$

Elimination of $\eta_{\gamma}$ from (4.9) and (4.10) gives

$$
M\left(\bar{\lambda}_{\gamma}-\lambda,_{\gamma}\right)+\lambda b_{\alpha \gamma}\left(\varrho_{2} U_{2}^{\alpha}-\varrho_{1} U_{1}^{\alpha}\right)=0 .
$$

Let us now define the strength of the shock, denoted by $\delta$, by the equation

$$
[\varrho]=\varrho_{1} \delta .
$$

The jumps in $V^{A}$ and $\mu$ can now be expressed in terms of $\delta$ as follows.

By using the last of Eqs. (4.4), rewrite (4.2) as

$$
\tau_{2}\left(-\lambda+V_{1}^{B} N_{B}\right)=M
$$

Therefore,

$$
\lambda=\left(\frac{\mu_{1}}{\varrho_{1}}-\frac{\mu_{2}}{\varrho_{2}}\right) M=\frac{M}{\varrho_{1}(1+\delta)}\left(\mu_{1}(1+\delta)-\mu_{2}\right) .
$$

It follows from (4.5) and (4.13) that

$$
\eta=c^{2} M^{2}\left(\frac{\mu_{1}}{\varrho_{1}}-\frac{\mu_{2}}{\varrho_{2}}\right) .
$$

It can also be shown that (4.3) leads to the equation

$$
c^{2}\left(\mu_{2}^{2}-\mu_{1}^{2}\right)=\left(\frac{\mu_{2}}{\varrho_{2}}+\frac{\mu_{1}}{\varrho_{1}}\right) \eta .
$$

Eliminate $\eta$ from (4.14) and (4.15) to obtain

$$
[\mu]=\mu_{1}\left(\frac{P_{1}(1+\delta)}{P_{2}}-1\right),
$$

where

$$
P_{1}=\left(\varrho_{1}^{2}+M^{2}\right)^{1 / 2}, \quad P_{2}=\left(\varrho_{2}^{2}+M^{2}\right)^{1 / 2} .
$$

By using the Eqs. (4.6), (4.13), and (4.16) in (4.11), we obtain

$$
\bar{\lambda}_{\gamma}-\lambda,_{\gamma}=-\frac{\left(P_{1}-P_{2}\right)^{2}}{P_{1} P_{2}} b_{\alpha \gamma} V_{1}^{\alpha} .
$$

The Eq. (4.17) is the basic result needed to obtain an expression for the jump in vorticity across the shock. 


\section{Jump in Vorticity}

Consider the vorticity vector $W^{A}$ defined by [9]

$W^{A}=\frac{1}{2} E^{A B C D} \omega_{C D} U_{B}$

where

$$
\begin{aligned}
& \omega_{C D}=U_{C, D}-U_{D, C} \\
& E^{A B C D}=\frac{1}{c} \varepsilon^{A B C D}, \quad E_{A B C D}=c \varepsilon_{A B C D}
\end{aligned}
$$

$\varepsilon_{A B C D}=\varepsilon^{A B C D}$ being permutation symbols with the usual meaning.

We now take jumps in (5.1) using (3.5), (4.1), and (4.4) and the fact that the state ahead of the shock is constant, to obtain

$$
\left[W^{A}\right]=\frac{1}{2 \mu_{2}^{2}} E^{A B C D} V_{B 2}\left\{\bar{\lambda}_{C} N_{D}-\bar{\lambda}_{D} N_{C}+a^{\alpha \beta} \lambda_{, \alpha}\left(N_{C} x_{D, \beta}-N_{D} x_{C, \beta}\right)\right\} .
$$

It follows immediately from (5.2) that

$$
\left[W^{A}\right] N_{A}=0,
$$

in view of the skew-symmetry of $E^{A B C D}$. That is, the normal component of the vorticity is continuous across the shock.

In order to obtain the jump in the tangential component of the vorticity vector, multiply (5.2) by $x_{A, \gamma}$ and sum over $A$, resolving the vector $\bar{\lambda}_{A}$ into components tangent and normal to the shock. In the resulting equation, use (4.16), the contravariant form of (4.17) and (4.1) to obtain the jump in the tangential component of vorticity vector across the shock as

$$
\left[W_{y}\right]=\frac{P_{2}}{P_{1}(1+\delta)^{2}}\left(1-\frac{P_{2}}{P_{1}}\right)^{2} E^{A B C D} x_{A, \gamma} U_{B 1} N_{C} x_{D, \alpha} b_{\beta}^{\alpha} U_{1}^{\beta} .
$$

It is clear from the Eqs. (5.3) and (5.4) that the jump in vorticity across a shock of given strength $\delta$, and of given curvature (i.e. $b_{\beta}^{\alpha}$ ) depends only on the velocity of the medium ahead. Note that in equation (5.4) although the velocity vector $U_{B 1}$ appears in addition to $U_{1}^{\beta}$, only its tangential component contributes, whereas the term involving its normal component vanishes in view of the skew-symmetry of $E^{A B C D}$.

Let us now discuss equation (5.4) in the rest frame of the shock surface $\Sigma$. If $G$ is the normal co-ordinate velocity of $\Sigma(t)$, considered as a moving surface in the $x^{i}$ space, and $n^{i}$ the unit normal to $\Sigma(t)$ in this space, then we have [7]

$$
N^{A}=\bar{\gamma}\left(\frac{G}{c^{2}}, n^{i}\right), \quad N^{A}=\bar{\gamma}\left(G,-n_{i}\right), \quad \bar{\gamma}^{2}=\left(1-G^{2} / c^{2}\right)^{-1} .
$$

Consider an orthonormal tetrad of vectors $N^{\prime A}, T_{(\alpha)}^{\prime A}(\stackrel{\circ}{\alpha}=0,1,2)$ at a point $P$ on the shock $\Sigma$. Then the transformation to this frame from a frame in which the shock has three-dimensional normal speed $G$ is given by

$$
\left.\begin{array}{ll}
L_{0}^{0}=\bar{\gamma}, \quad L_{0}^{i}=\bar{\gamma} G n^{i} & L_{i}^{o}=\frac{\bar{\gamma} G n_{i}}{c^{2}} \\
L_{i}^{j}=\delta_{i}^{j}+(\bar{\gamma}-1) n_{i} n^{j}, &
\end{array}\right\}
$$


so that any vector $Q^{A}$ transforms according to

$$
Q^{\prime A}=h^{A B} L_{B}^{C} Q_{C} .
$$

In particular, we have

$$
N^{\prime A}=\left(0, n^{i}\right), T_{(0)}^{\prime A}=\left(\frac{1}{c}, 0\right), T_{(1)}^{\prime A}=\left(0, t_{(1)}^{i}\right), T_{(2)}^{\prime A}=\left(0, t_{(2)}^{i}\right) .
$$

By using (5.6) and (5.8) it can be shown that, at the point $P$, the components $b_{0 \alpha}$ vanish. Further, let us assume that $b_{12}=b_{21}=0$ at the point $P$. By setting $\left[\Omega_{\gamma}\right]=$ $c\left[W_{\gamma}^{\prime}\right]$, and using (5.6) - (5.8), the space components of (5.4) in the rest frame of the shock can be obtained as

$$
\begin{aligned}
& {\left[\Omega_{(1)}\right]=Y \gamma^{2} \bar{\gamma}\left(1-\frac{G v_{n}}{c^{2}}\right) v_{(2)} b_{2}^{2}, \quad \gamma^{2}=\left(1-v^{2} / c^{2}\right)^{-1}, \quad v^{2}=v^{i} v_{i},} \\
& {\left[\Omega_{(2)}\right]=-Y \gamma^{2} \bar{\gamma}\left(1-\frac{G v_{n}}{c^{2}}\right) v_{(1)} b_{1}^{1},}
\end{aligned}
$$

where $Y=P_{2}\left(P_{1}-P_{2}\right)^{2} /\left(P_{1}^{3}(1+\delta)^{2}\right), v_{n}=v^{i} n_{i}, v^{i}$ being the ordinary velocity vector of the fluid, and the brackets around subscripts indicate the surface components. The time component $\left[\Omega_{(0)}\right]$, which is not independent of $\left[\Omega_{(1)}\right]$ and $\left[\Omega_{(2)}\right]$ can be obtained either directly from (5.4) or by using the fact $\left[W_{A} V^{A}\right]=0$, and the Eqs. (4.6) and (4.7).

By letting $c \rightarrow \infty$ in (5.9), we obtain the classical case

$$
\left[\Omega_{(1)}\right]=\delta^{2} /(1+\delta) v_{(2)} d_{2}^{2}, \quad\left[\Omega_{(2)}\right]=-\delta^{2} /(1+\delta) v_{(1)} d_{1}^{1} .
$$

Comparing (5.9) with (5.10), apart from the factor $Y$ whose limiting value is $\delta^{2} /(1+\delta)$, the contraction factors $\gamma, \bar{\gamma}$ appear in the relativistic expressions, as one would expect.

Acknowledgement. The author wishes to thank Dr. G. A. Nariboli, Professor, Department of Engineering Science and Mechanics, Iowa State University, Ames, Iowa, USA, for his suggestions and help in this work. The author is also grateful to Professor J. Ehlers, Max-Planck-Institut für Physık und Astrophysik, München, West Germany, for his invaluable suggestions on an earlier version of this paper.

\section{References}

1. Taub, A.H.: Phys. Rev. 74, 328-334 (1948)

2. Johnson, M.H., McKee, Ch.F.: Phys. Rev. D. 3, 858-863 (1971)

3. Truesdell, C.: J. Aero. Sci. 19, 826-828 (1952)

4. Lighthill, M. J.: J. Fluid Mech. 2, 1-32 (1957)

5. Hayes, W.D.: J. Fluid Mech. 2, 595-600 (1957)

6. Taub, A. H.: Phys. Rev. 74, 328-334 (1948)

7. Thomas, T. Y.: J. Math. Anal. Appl. 7, 225-246 (1963)

8. Nariboli, G. A.: Tensor, N.S. 20, 161-166 (1969)

9. Taub, A.H.: Arch. Rat. Mech. Anal. 3, 312-324 (1959) 